\title{
Characterization of a Laser-Generated Perturbation in High-Speed Flow for Receptivity Studies
}

\author{
Amanda Chou* \\ School of Aeronautics \&3 Astronautics, Purdue University, West Lafayette, IN 47907, USA \\ NASA Langley Research Center, Hampton, VA 23681 \\ Steven P. Schneider ${ }^{\dagger}$ \\ School of Aeronautics 83 Astronautics, Purdue University, West Lafayette, IN 47907, USA \\ Michael A. Kegerise ${ }^{\ddagger}$ \\ NASA Langley Research Center, Hampton, VA 23681
}

\begin{abstract}
A better understanding of receptivity can contribute to the development of an amplitudebased method of transition prediction. This type of prediction model would incorporate more physics than the semi-empirical methods, which are widely used. The experimental study of receptivity requires a characterization of the external disturbances and a study of their effect on the boundary layer instabilities. Characterization measurements for a lasergenerated perturbation were made in two different wind tunnels. These measurements were made with hot-wire probes, optical techniques, and pressure transducer probes. Existing methods all have their limitations, so better measurements will require the development of new instrumentation. Nevertheless, the freestream laser-generated perturbation has been shown to be about $6 \mathrm{~mm}$ in diameter at a static density of about $0.045 \mathrm{~kg} / \mathrm{m}^{3}$. The amplitude of the perturbation is large, which may be unsuitable for the study of linear growth.
\end{abstract}

\section{Nomenclature}

diameter, $\mathrm{mm}$ voltage, $\mathrm{V}$ frequency, $\mathrm{Hz}$ or $\mathrm{kHz}$ ratio of aperture to focal length Mach number pressure, $\mathrm{kPa}$ temperature, $\mathrm{K}$ streamwise velocity, $\mathrm{m} / \mathrm{s}$ horizontal location, mm (along optical axis) vertical location, $\mathrm{mm}$ (perpendicular to optical axis) distance from nozzle throat, $\mathrm{m}$ distance between probe and perturbation

$$
\rho \quad \text { density, } \mathrm{kg} / \mathrm{m}^{3}
$$

Subscript

0 stagnation condition

1 in front of a shock

2 behind a shock

$i \quad$ initial condition

meas measurement location

n.e. $\quad$ at the nozzle exit

perturb location of perturbation generation $\infty \quad$ in the freestream
Superscript

fluctuation

*Graduate Research Assistant and Student Intern, AIAA Student Member.

${ }^{\dagger}$ Professor, AIAA Associate Fellow.

${ }^{\ddagger}$ Research Scientist, Flow Physics \& Control Branch, AIAA Member. 


\section{Introduction}

Laminar-turbulent transition in the hypersonic regime is not a well-understood process. To understand this phenomenon, experimental data are needed. Flight tests cost orders of magnitude more than ground tests and generally return less data, so experiments conducted in wind tunnels are required. Receptivity, as termed by Morkovin in his 1969 review of transition, ${ }^{1}$ is the process by which freestream disturbances enter the boundary layer. When these disturbances enter the boundary layer, they can excite instabilities. These instabilities can affect the transition process by growing to create large fluctuations in the boundary layer, which can eventually break down into turbulence.

Current high-speed transition prediction methods are semi-empirical at best and incorporate only some physics. These methods estimate the transition process by examining the relative growth of instabilities. The initial amplitude of the instability may not be fully considered in a semi-empirical prediction method. Instead, the transition criterion is changed depending on the environment or model. Choosing a transition criteria based only on the relative growth of instabilities may not take into consideration that the initial amplitude of the instability may be very large or very small. Receptivity studies can be used to help determine the initial amplitudes of the instabilities. If the receptivity process can be better understood, then a finite-amplitude-based method of transition prediction can be developed.

In a previous study, Mack developed a forcing theory ${ }^{2}$ to apply to flat plate measurements ${ }^{3}$ taken at the Jet Propulsion Lab (JPL) in Pasadena, CA. This theory uses a forced response of the boundary layer to predict the growth of instabilities. The forcing theory was found to provide computations in good agreement with the measurements. However, the forced disturbances were distinctly different from the freestream disturbances. The mechanism by which the freestream disturbances become forced disturbances was not described with this theory, as Mack noted in Reference. ${ }^{2}$ This disconnect remains an ongoing problem, and many measurements and computations need to be done to reconcile this gap.

Past computational and theoretical receptivity studies have included investigations of acoustic waves ${ }^{4-6}$ of vorticity waves, ${ }^{7}$ of discrete particles, ${ }^{8}$ and of small entropic disturbances. ${ }^{9,10}$ One of the largest problems in experimental receptivity studies lies in the ability to create well-defined, well-controlled disturbances. First, the disturbance must be repeatable and its amplitude and frequency content well-controlled. Second, the disturbance must not be so large that the freestream disturbance causes bypass transition. Lastly, the equipment used to generate the upstream disturbance must not alter the overall freestream conditions (e.g., mean background noise level, Mach number, etc.). Experimental studies have largely been in the subsonic regime, with disturbances that are difficult to characterize, such as those created by jets ${ }^{11}$ and speakers. ${ }^{12}$ The few high-speed studies of the receptivity to freestream disturbances have included the use of an upstream electrode to generate acoustic disturbances ${ }^{13}$ and the use of a high-powered laser to generate a thermal disturbance. ${ }^{14-16}$

The method of creating freestream disturbances with a high-powered laser was first developed at Purdue University in the 1990s. ${ }^{14,17}$ The effect of this disturbance on the boundary layer instabilities is studied to understand how disturbances first enter the boundary layer and cause transition. Previous receptivity research using the laser-generated perturbation has been conducted by Schmisseur on an elliptic cone, ${ }^{18}$ and later by Heitmann on a flat plate ${ }^{16}$ and a slender cone. ${ }^{19,20}$ Schmisseur found that the thermal perturbation generated in the freestream was too large to be measured by a hot wire. No instability waves were measured on a 4:1 elliptic cone model with this perturbation at Mach-4 in the old Purdue Quiet Flow Ludwieg Tube. ${ }^{18}$ Heitmann's experiments use the acoustic disturbance generated by the laser-induced breakdown process. This acoustic disturbance is much weaker than the thermal disturbance. Furthermore, due to the limitations in optical access, the disturbance is generated aft of the model's shock wave. ${ }^{16,21}$

Improvements on these experiments are desired in order to contribute to an amplitude-based model for transition prediction. To do so, the freestream disturbance must be characterized quantitatively using measurements with probes or other techniques. Without an understanding of the freestream disturbances present, it is difficult to develop models of how these disturbances affect the instability growth and transition process. Measurements shown in this paper are used to define and characterize the laser-generated perturbation for later receptivity studies. Ideally, both amplitude and frequency content would be obtained from these measurements. Hot wire probes, optical techniques, and fast pressure transducers are used in two different tunnels to attempt to provide quantitative dynamic measurements of the freestream perturbation. However, these measurement techniques are limited in terms of either bandwidth, spatial resolution, electronic noise, or a combination of all of the above. Some of these instrumentation choices, such as hot wires and pitot probes, may not be suited to the measurement of a thermal disturbance, as they are sensitive to 
other physical characteristics. Nevertheless, characteristics of the perturbation can still be determined, even if they are at best, only qualitative.

\section{Facilities and Apparatus}

\section{A. Purdue University Boeing/AFOSR Mach-6 Quiet Tunnel (BAM6QT)}

The Boeing/AFOSR Mach-6 Quiet Tunnel (BAM6QT) is currently one of three operational hypersonic quiet tunnels in the world (Fig. 1). Quiet tunnels have laminar nozzle-wall boundary layers. Conventional tunnels have turbulent nozzle-wall boundary layers, which radiate acoustic noise into the freestream of the tunnel. This creates a high-noise environment that is orders of magnitude more than actual flight conditions. ${ }^{22,23}$ Laminar boundary layers on the nozzle wall are capable of providing ground test environments that have pressure fluctuations similar to what is seen in flight.

Several features are used to maintain laminar boundary layers on the nozzle wall. Filters remove particles from the freestream to minimize the likelihood of particle impact. A mirror-finish polish on the nozzle wall reduces the possibility of roughness-induced transition. The nozzle is also longer than that of a conventional tunnel to minimize the likelihood of transition due to the Görtler instability. A bleed valve suctions off the air at the throat, allowing a fresh boundary layer to grow along the length of the nozzle. To run this tunnel in a conventional or "noisy" configuration, the bleed valve is simply closed off to trip the boundary layer.

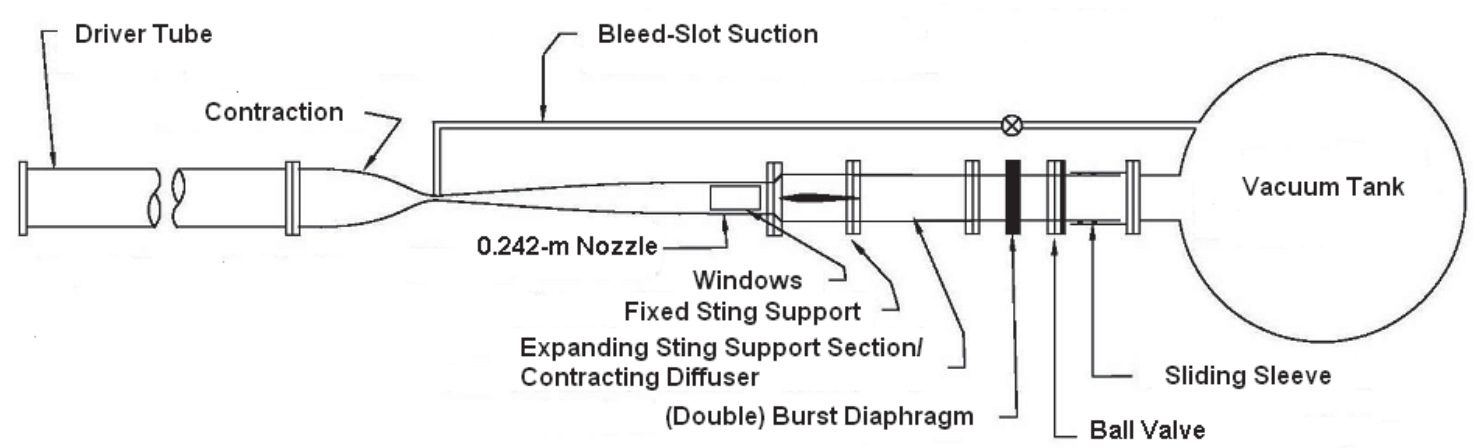

Figure 1. Schematic of the Boeing/AFOSR Mach-6 Quiet Tunnel (BAM6QT)

The BAM6QT is a Ludwieg tube with a double-burst diaphragm system downstream of the test section, so models and instrumentation must be able to withstand the full stagnation pressure. Due to this configuration, the tunnel conditions also experience a stair-step decrease every 0.2 seconds, as the expansion wave reflects between the end of the driver tube and throat. When the tunnel is run in a "quiet" configuration, the facility also experiences an increase in noise approximately 2 seconds after startup. ${ }^{24}$ However, at lower stagnation pressures, this increase in noise may still be less than $0.1 \%$ of the freestream.

The survivability of delicate sensors such as hot wires is fairly low in this facility, due to the violent start-up and shut-down processes. Probes using robust sensors are more commonly used instead. The measurements taken in this facility and presented in this paper use only pressure transducer probes.

Previous measurements of the flow quality in the BAM6QT show low acoustic noise up to stagnation pressures greater than $1150 \mathrm{kPa}^{24}$ The uniformity of the flow was previously investigated by Steen and found to be consistent across a variety of pressures and measurement locations. ${ }^{24,25}$ Modifications to the tunnel were required in order to use perturbation-forming optics. The effects of this configuration were investigated and presented in a previous paper. ${ }^{26}$ The flow is still expected to be quiet at the measurement locations presented in this paper in the modified configuration. The measurements presented in Reference 26 show an acoustic noise level of about $0.03 \%$ at a location of $50-60 \mathrm{~mm}$ off the centerline at an axial location of $z=2.374 \mathrm{~m}$. 


\section{B. NASA Langley Research Center Probe Calibration Tunnel (PCT)}

The Probe Calibration Tunnel (PCT) is a conventional facility located at the NASA Langley Research Center (LaRC), which is capable of reaching a Mach number of 3.5. This facility is primarily used for sensor calibrations. The tunnel is a blow-down tunnel, which can be run continuously with the infrastructure available at NASA LaRC. The pressure and temperature of this facility can be varied independently.

The long-duration runs of this tunnel allow for very detailed measurements of the perturbation. Furthermore, the open-jet configuration of the tunnel allows for the hot wires to be shielded from the flow during the start-up and shut-down processes, which is when many wires break. This is not possible in the BAM6QT. However, the PCT is smaller in size, having a 8-cm-diameter nozzle compared to the BAM6QT's 24.2-cm-diameter nozzle. A probe can be mounted to a probe holder strut, as shown in Figure 2. This probe holder can traverse in the $\pm x$-direction, horizontally across the nozzle exit of the PCT (Figure 3 ).

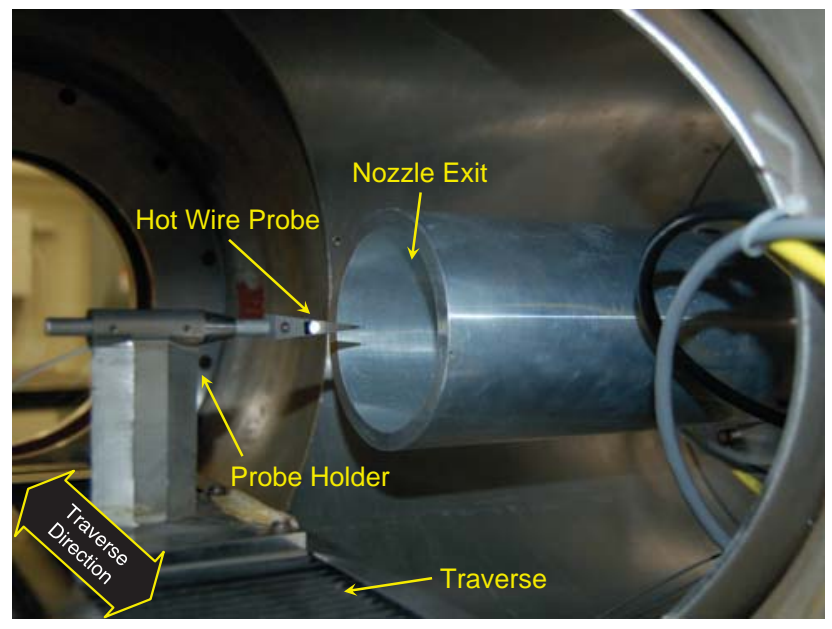

Figure 2. Test section of PCT, showing setup with traverse and hot wire probe.

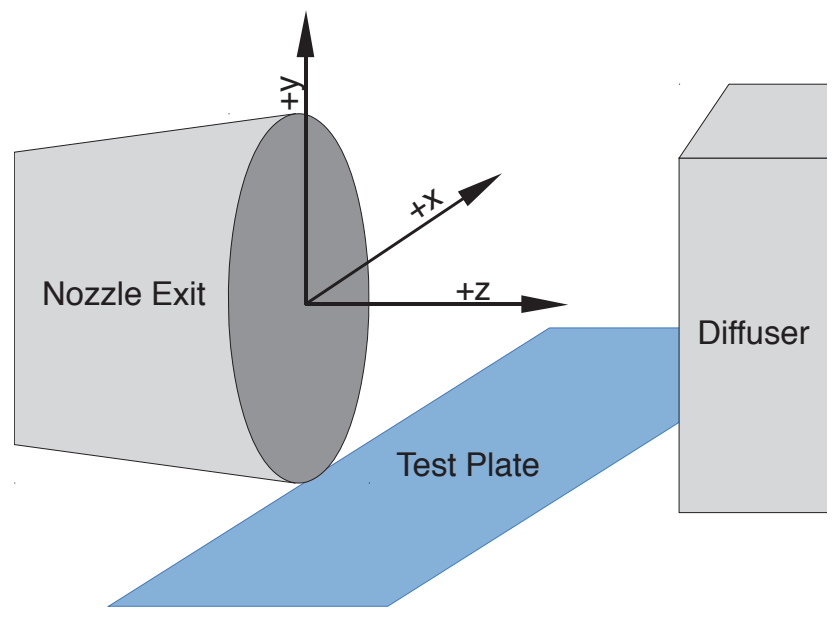

Figure 3. Schematic of test section of PCT, showing coordinate system.

The PCT was not designed as a quiet facility, and thus, may have higher acoustic noise than the BAM6QT. However, hot-wire measurements in the freestream indicate broadband levels of fluctuation indicative of quiet flow at the stagnation pressures tested. These tests are discussed later in Section IV.B. 


\section{A Comparison of Facilities}

A comparison of the conditions in the different tunnels is provided in Table 1. If the disturbance is estimated to be about $2 \mathrm{~mm}$ in diameter $(D),{ }^{18}$ then the frequency response required to resolve this perturbation in the BAM6QT is on the order of $1 \mathrm{MHz}$. Few types of instrumentation have this frequency response. Optical measurement techniques are the primary candidate. Characterization measurements in a facility with a lower freestream velocity require less bandwidth. Thus, it may be desirable to attempt to characterize the perturbation at a lower velocity first. Another advantage of using a facility such as the LaRC PCT is the benefit of longer run times. A long run time allows for detailed measurements that would be difficult to make in a facility such as the BAM6QT.

Table 1. A comparison of conditions and disturbance speeds in three tunnels.

\begin{tabular}{lcc}
\hline & BAM6QT & PCT \\
\hline \hline Nominal Mach Number & 6.0 & 3.5 \\
\hline Stagnation Temperature $T_{0}, \mathbf{K}$ & 433 & 311 \\
\hline $\begin{array}{l}\text { Freestream Speed } u_{\infty}, \mathbf{m} / \mathbf{s} \\
\text { (Speed of Thermal Disturbance) }\end{array}$ & 874 & 660 \\
\hline Frequency Response Required $2 u_{\infty} / D, \mathbf{k H z}$ & 874 & 660 \\
\hline
\end{tabular}

The perturbation created in different facilities with different equipment may not be the same. Direct comparisons of the measurements made in the BAM6QT and PCT are required. To compare the measurements in these different facilities, the freestream static density in each facility is matched. This assumes that the generation and development of the perturbation in the freestream is largely driven by the density rather than other flow characteristics, such as Reynolds number. The corresponding stagnation pressure and temperature for both tunnels at each of the desired densities is provided in Table 2.

Table 2. BAM6QT and PCT conditions corresponding to densities where a laser perturbation was created and measured.

\begin{tabular}{cccccc}
\hline \multirow{2}{*}{ Case } & Density & \multicolumn{2}{c}{ BAM6QT $\left(M_{\infty}=6.0\right)$} & \multicolumn{2}{c}{ PCT $\left(M_{\infty}=3.5\right)$} \\
\cline { 5 - 6 } & $\rho_{\infty}, \mathbf{k g} / \mathbf{m}^{3}$ & $p_{0}, \mathbf{k P a}$ & $T_{0}, \mathbf{K}$ & $p_{0}, \mathbf{k P a}$ & $T_{0}, \mathbf{K}$ \\
\hline \hline BAM6QT Max. Quiet & 0.049 & 1170 & 433 & 96.7 & 311 \\
\hline $\begin{array}{c}\text { Transitional Boundary Layer } \\
\text { in Quiet Flow on a Flared Cone }\end{array}$ & 0.043 & 1030 & 433 & 85.3 & 311 \\
\hline Intermediate Condition & 0.035 & 827 & 433 & 68.2 & 311 \\
\hline $\begin{array}{c}\text { Laminar Boundary Layer } \\
\text { in Quiet Flow on a Flared Cone }\end{array}$ & 0.026 & 621 & 433 & 51.2 & 311 \\
\hline
\end{tabular}

\section{The Laser-Generated Perturbation}

The perturbation used for these receptivity studies is created by focusing a high-powered Nd:YAG laser to a small region in the freestream of a wind tunnel. This creates a laser-induced breakdown plasma in the freestream. This plasma quickly cools and a weak shock wave propagates from this thermal core. This process is detailed in several referemces, including References 28-30. The weak shock wave dissipates and the thermal perturbation persists to interact with downstream models or probes. A schematic of the structure of this perturbation is provided in Figure 4. The thermal core and shock wave should ideally be spherical.

\section{Nd:YAG Laser}

The laser used with the BAM6QT is a Spectra-Physics GCR-190 with a 4-mm beam diameter. The laser used with the PCT is a Spectra-Physics PIV-400 laser with a 9-mm beam diameter, which has been reduced to a 4-mm beam diameter by an aperture. Although the PIV-400 is a double-pulse laser, only the first pulse is used to generate perturbations in the freestream of the flow. Both lasers are frequency-doubled Nd:YAG 


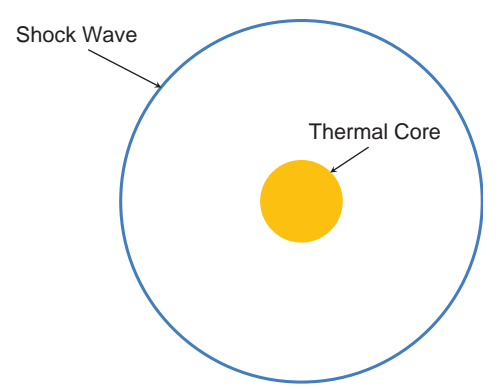

Figure 4. Schematic of the composition of the ideal laser-generated perturbation.

lasers, emitting light at $532 \mathrm{~nm}$. These lasers are also both seeded, and pulse at $10 \mathrm{~Hz}$, with a pulse width of about $7 \mathrm{~ns}$.

\section{Forming Optics}

The laser-generated perturbation is created by focusing a high-powered laser down to a small volume. This is accomplished using three lenses (Figure 5):

1. a CVI/Melles-Griot YAN-50.0-10.0 lens to expand the beam diameter

2. a CVI/Melles-Griot YAP-200.0-40.0 lens to collimate the beam

3. a CVI/Melles-Griot YAP-200.0-40.0 lens to focus the beam

The three-lens system increases the $f / D$ ratio of the optics, which ensures as small a focal volume as possible. A smaller focal volume increases the energy density of the system and allows for perturbations to be made more easily in low-density environments, such as a wind tunnel.

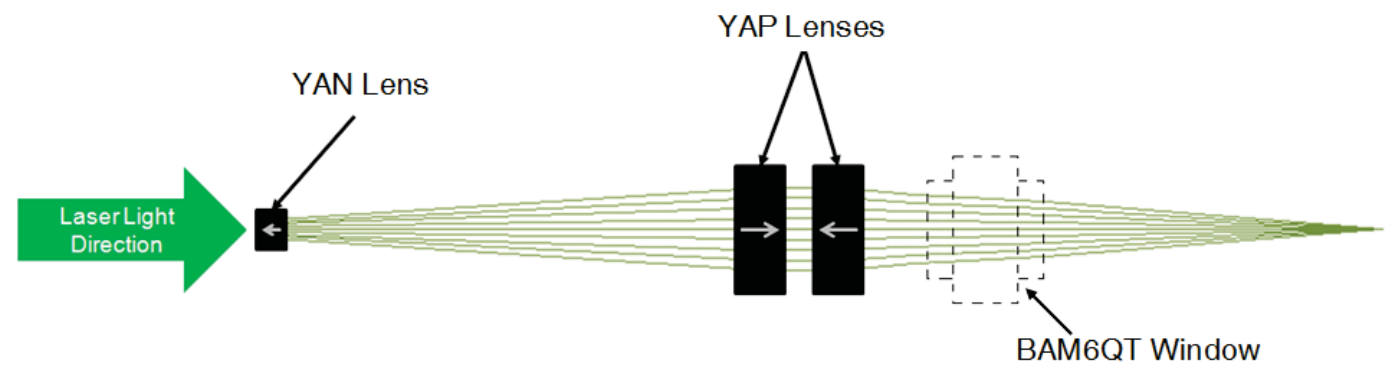

Figure 5. Schematic of the optical setup. Laser light propagates from left to right.

Hot-wire measurements in the PCT were conducted with only the last YAP-200.0-40.0 lens in the system. This was done because a method of fitting the BAM6QT optics in the PCT had not yet been devised. The effect of using this modified optical system is that the beam diameter is reduced and the $f / \#$ is increased. This effectively reduces the converging angle of the focused light and causes the focal region to become elongated and more elliptical. This could cause the breakdown process to require higher energy in order to achieve breakdown and could possibly distort the initial shape of the perturbation. However, measurements are typically taken farther downstream, so this optical modification may have little effect. Deflectometry tests in the PCT use the BAM6QT optics, so that both tunnels use the same perturbation-forming optics.

\section{Methods}

\section{A. Measurements in the PCT}

A Mensor Model 2101 pressure gauge is used to measure the stagnation pressure of the PCT during the run. A resistance temperature detector (RTD) connected to a digital multimeter is used to measure the stagnation temperature. Changes in the stagnation pressure cause changes in the displacement thickness, which can change the Mach number at the nozzle exit. Thus, a "Mach number calibration" is required. 
The Mach number is inferred from measurements of the pitot pressure, using the Rayleigh pitot formula in a separate calibration run. For this calibration run, a pitot probe on the centerline is used to measure the pitot pressure. The Mach number of the flow at each hot wire calibration point is found using a 1-D lookup table which uses this stagnation-pressure-to-Mach-number calibration.

\section{Hot-Wire Measurements}

A TSI IFA-100 constant temperature anemometer was used with a 5 - $\mu$ m-diameter platinum-plated tungsten wire. The wire aspect ratio was at least 250 to minimize end-conduction effects. The hot wire was tuned to have a frequency response of about $260 \mathrm{kHz}$, and the signal was sampled at $1 \mathrm{MHz}$. A 1:1 bridge is used for these measurements. The overheat ratio, $\tau$, is defined as:

$$
\tau=\frac{T_{w}-\eta T_{0}}{T_{0}}
$$

where $T_{w}$ is the wire temperature and $\eta=0.94$ is the wire recovery factor found from previous tests by Kegerise et al. ${ }^{31}$ The overheat ratio used for these tests was between 0.75 and 0.80 , to bias the hot wire toward a higher sensitivity to mass flux variation. ${ }^{32}$

A calibration of the wire was taken by holding the probe on the nozzle centerline while varying the stagnation pressure over a range of values. The stagnation temperature was held constant at $310 \pm 1.5 \mathrm{~K}$. The hot-wire voltage was sampled at each condition for the calibration. A calibration curve of the form:

$$
E_{0}^{2}\left(\frac{T_{0}}{T_{c}}\right)=L+M(\rho u)^{n}
$$

was assumed as in Reference 31 . Here, $E_{0}$ is the mean voltage output, $T_{c}=310 \mathrm{~K}$ is the nominal calibration temperature, and $\rho u$ is the mass flux. This form of King's Law assumes that the temperature fluctuations are fairly small. In addition, this calibration is only applicable at high overheat ratios and within a temperature range of $\pm 20 \mathrm{~K}$. The constants $L, M$, and $n$ are found using a Gauss-Newton nonlinear least-squares method.

The hot wire calibration is used only for making freestream mass-flux measurements. The large temperature change across the laser-generated thermal perturbation invalidates the assumptions of the calibration. Thus, measurements of the thermal perturbation with this technique are only qualitative in nature.

Since the data were taken in an open jet, the inferred mass flux was then normalized by the nominal mass flux, or mass flux at the nozzle exit. The nominal mass flow $\left(\rho u_{n . e .}\right)$ is then calculated using the isentropic flow relations for a perfect gas to give the equation:

$$
\rho u_{n . e .}=\frac{p_{0}}{\sqrt{T_{0}}} \sqrt{\frac{\gamma}{R}} M\left(1+\frac{\gamma-1}{2} M^{2}\right)^{-\frac{\gamma+1}{2(\gamma-1)}}
$$

where $p_{0}$ is the stagnation pressure, $\gamma$ is the ratio of specific heats and assumed to be 1.4 , and $R$ is the gas constant $287 \mathrm{~J} /(\mathrm{kg} \cdot \mathrm{K})$. In all of the hot-wire measurements following, $z$ is the streamwise distance from the nozzle exit and $x$ is the horizontal distance from the centerline. The positive- $x$ direction corresponds to a direction toward the laser head and the negative- $x$ direction corresponds to a direction away from the laser head.

\section{Deflectometry Measurements}

An optical deflectometry method was used to characterize the laser-generated perturbation at higher frequencies. The deflectometry method used in this experiment is similar to that used by McIntyre et al. ${ }^{33}$ The method used by McIntyre et al. involves the use of a schlieren system with a continuous light source. In this case, the light source used was a xenon arc lamp. Variations in the light intensity and in the room temperature are accounted for by referencing measurements taken within the flow field to one taken outside of the flow field. Fiber optic cables can be made to traverse the schlieren image. These fiber optic cables can be connected to photomultiplier tubes in order to convert the illumination levels at a point on the schlieren image to an analog signal.

The schlieren system used in the present experiment uses a Toepler Z-type setup. The light source is collimated with a 50-inch-focal-length parabolic mirror to fully illuminate the test section. The light passing through the test section is focused with another 50-inch-focal-length parabolic mirror to a knife edge. A 
horizontal knife edge is oriented to make the measurements sensitive to the density fluctuation normal to the flow. In this setup, it is assumed that the flow travels exactly parallel to the horizontal axis. The resulting image with cutoff was split with a 50/50 beamsplitter immediately after the knife edge to allow for simultaneous measurements at two different locations. These two images are referred to as a "reference" image, which is measured with a fixed fiber, and a "probe" image, which is measured with a traversing fiber. The reference measurements are taken with a fixed fiber to account for small fluctuations in light intensity and ambient temperature.

Two 200- $\mu \mathrm{m}$ fiber optic cables were used to probe the reference image and the probe image. The reference sensor used was a Hamamatsu H5784-20 photosensor module, which has a frequency response of $20 \mathrm{kHz}$. Only the mean voltage was used from this sensor. The probe sensor used was a Hamamatsu HC124-01 photomultiplier tube, which has a frequency response of $8 \mathrm{MHz}$. This high frequency response is expected to provide the necessary resolution to show the streamwise extent of the perturbation.

A calibration of the photosensors requires the matching of the deflection of a light beam across the knife edge to the voltage output of the photosensor. This can be obtained by simply traversing the knife edge across the image of the light source at the focal plane. The position of the knife edge corresponds to the vertical deflection of a light beam being measured at a certain probe location. This can then be correlated to an angular deflection using the focal length of the parabolic mirrors.

The deflection measured by the photosensors at a given point is the result of an integration across the perturbation. Previous measurements at Purdue by Schmisseur ${ }^{18}$ show that this perturbation is fairly symmetric. Assuming that the perturbation in the PCT is also axisymmetric, an inverse Abel transform can be used to convert the deflectometry measurements across the perturbation to a radial index of refraction. ${ }^{34}$ If the number of free electrons and ions in the perturbation is also assumed to be negligible, the index of refraction can be converted to a density via the Gladstone-Dale relation,

$$
n-1=K \rho
$$

where $n$ is the index of refraction, $K=2.259 \times 10^{-4} \mathrm{~m}^{3} / \mathrm{kg}$ for air, and $\rho$ is the density. The assumption of negligible free electrons and ions may be a poor assumption, however, considering that the lifetime of a laser-generated plasma can be up to some microseconds. The presence of the free electrons and molecular ions can also last for several tens of microseconds. In the PCT, this translates to a distance on the order of about $50 \mathrm{~mm}$ downstream of the perturbation generation location.

\section{B. Probe Measurements in the BAM6QT}

A laser perturbation is made in the freestream of the BAM6QT. This is aligned to the centerline of the tunnel using a probe alignment cap, which attaches to the tip of a pressure sensor probe. The coordinate system used for measurements in the BAM6QT is the same as that used in the PCT (Figure 3), for consistency. Probes in the BAM6QT can only traverse in the $y z$-plane, and only along the $y$-axis during a run. The $z$-position of the probe can be adjusted between runs. The stagnation pressure is measured with a Kulite XTEL-190-500A transducer mounted in the driver tube. The stagnation temperature is measured with a K-type Omega thermocouple fed to an Omega DP18 meter.

\section{Kulite Probe Measurements}

A Kulite XCQ-062-15A pressure sensor with a B-screen is used for measurements in the BAM6QT. This screen further reduces the frequency response of the sensor, but a B-screen helps to protect the sensor from particle impact when it is in a forward-facing configuration. Although there are few particles in the BAM6QT, there may still be small particles that could destroy the sensor. The diameter of the XCQ-062 sensor is about $1.59 \mathrm{~mm}$, which is on the order of the expected size of the laser-generated disturbance, so some spatial averaging is expected.

A Kulite sensor can be mounted to a traversing probe in the BAM6QT. The frequency response of a Kulite pressure transducer is about an order of magnitude less than the predicted frequency response required to resolve the thermal perturbation (Table 1). Kulite reports that the frequency response is only about $20 \mathrm{kHz},{ }^{35}$ but tests by Rotea ${ }^{36}$ and later by Beresh ${ }^{37}$ indicate that the frequency response may be as much as $20 \%$ of the resonant frequency of the sensor diaphragm. For the Kulite sensors used in these probe measurements, this benchmark implies a frequency response of up to $50-60 \mathrm{kHz}$. 


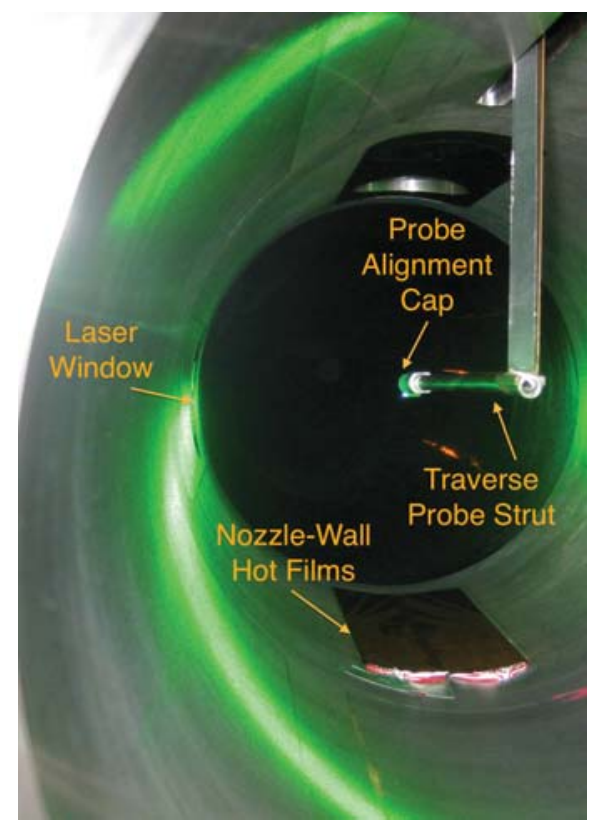

Figure 6. Alignment of pitot probe with alignment cap. (Looking upstream of the BAM6QT.)

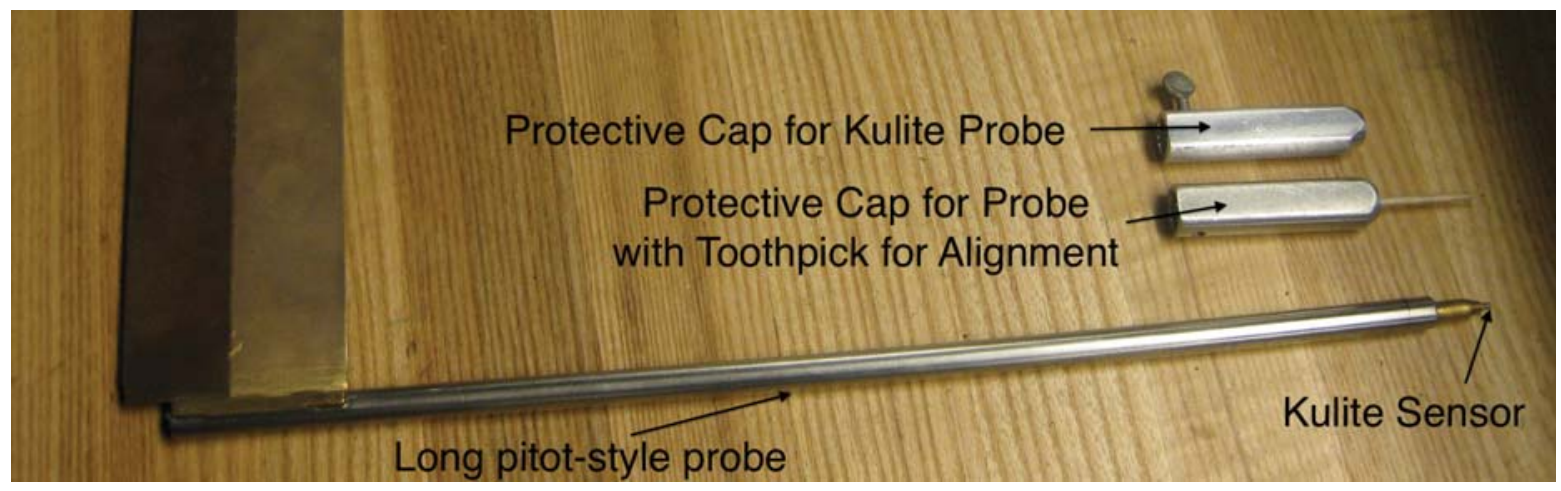

Figure 7. Long Kulite pitot probe with alignment cap and protective cap.

\section{2. $P C B$ Probe Measurements}

A PCB 132A31 fast pressure sensor can also be mounted to a probe, which is similar to that used for the Kulite sensors. The PCB 132A31 sensor has a 3.18-mm diameter and a reported frequency response of over $1 \mathrm{MHz}$. The subsonic region of this probe is much larger than that of a Kulite pressure transducer, which may have some effect on the measurements made with this probe. The sensor output, however, is high-pass filtered through signal conditioners with a $3-\mathrm{dB}$ cutoff at $11 \mathrm{kHz}$. These cutoff values mean that the PCB sensor provides only a measurement of pressure fluctuation. Thus, the mean pitot pressure must be inferred from the freestream stagnation pressure using the relation for the pressure across a normal shock wave:

$$
\frac{p_{0,2}}{p_{0,1}}=\left[\frac{(\gamma+1) M^{2}}{(\gamma-1) M^{2}+2}\right]^{\frac{\gamma}{\gamma-1}}\left[\frac{\gamma+1}{2 \gamma M^{2}-\gamma+1}\right]
$$

where $p_{0,2}$ is the pitot pressure, $p_{0,1}$ is the freestream stagnation condition, $\gamma=1.4$ is the ratio of specific heats, and $M$ is the freestream Mach number. Calibration of these sensors is not straightforward. Due to the lack of a mean voltage output from these sensors, a static calibration cannot be performed. Work is underway to devise methods of dynamic calibration. ${ }^{37,38}$ Thus, the factory-provided calibration is used for these pressure transducers. 
This sensor is a piezoelectric quartz sensor, which was originally manufactured as a time-of-arrival sensor. However, these sensors have shown an ability to measure high-frequency pressure fluctuations in hypersonic wind tunnels. ${ }^{39-42}$ The sensing element is a $0.762-\mathrm{mm}$ square placed arbitrarily on the head of the sensor. The surface of the sensor is coated with a conductive epoxy, which aids in the measurement of pressure. This makes the active sensing area uncertain, so the extent of spatial averaging is unknown.

\section{Contour Plots from Survey Data}

Contour plots are created by compiling data from a survey of the region through which the perturbation passes. The probe is held steady at each spanwise measurement location for some time. Blocks of data are taken after each laser pulse for several laser pulses. These blocks are then averaged together to reduce the background (electronic and freestream) noise. This requires the assumption that the laser perturbation is repeatable, since each time block is taken after a different laser pulse.

The pulse-to-pulse repeatability of the response to a laser-generated perturbation is fairly good, with less than $5 \%$ variation in the measured amplitude. The perturbation has been found repeatable in previous studies by Schmisseur ${ }^{18}$ and Salyer. ${ }^{15}$ Repeatability of the laser perturbation generated in both the BAM6QT and the PCT is illustrated by Figure 8. Figure 8(a) shows the normalized response of a Kulite probe in the BAM6QT on the centerline of the tunnel. Traces from 10 different laser shots are shown here. The response due to each laser shot is in a different color, but can barely be distinguished apart from one another due to the small amount of variation. This variation may be due mostly to the changes in freestream conditions as the expansion wave reflects in the driver tube. Figure 8(b) shows the response of a hot wire in the PCT on the centerline of the tunnel. Traces from 10 different laser shots are also shown here and shows as little variation as the Kulite measurements in the BAM6QT.

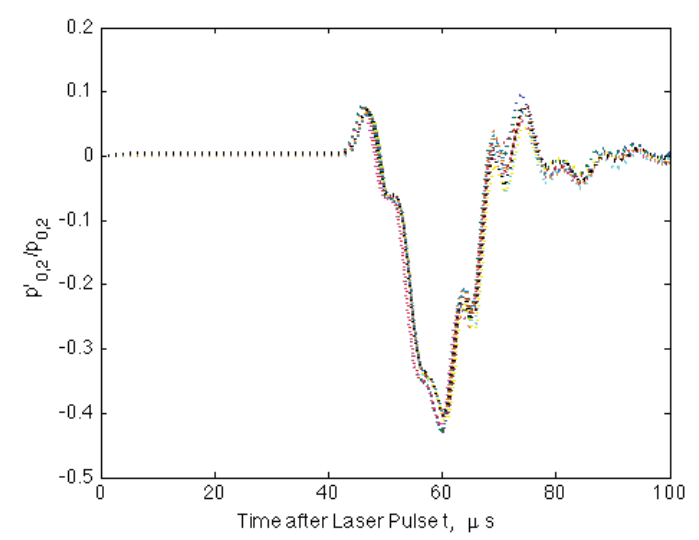

(a) Kulite measurement in BAM6QT. $p_{0, i}=1133 \mathrm{kPa}$, $T_{0, i}=435.7 \mathrm{~K}, M=6.0$, quiet.

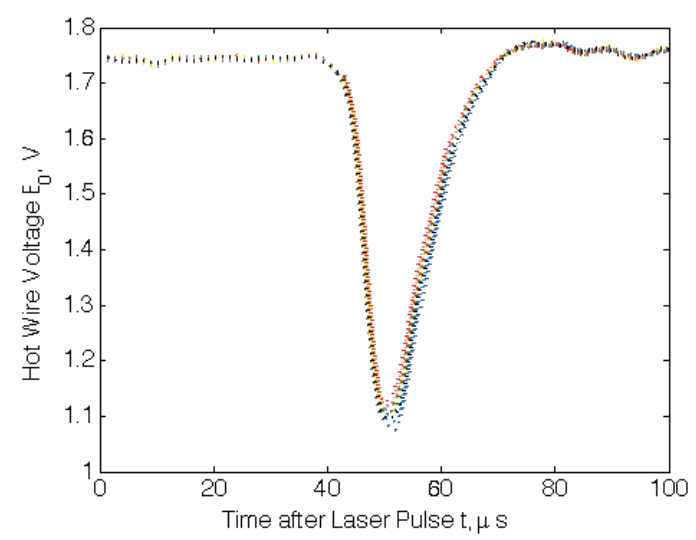

(b) Hot-wire measurement in PCT. $p_{0}=96.7 \mathrm{kPa}, T_{0}=$ $311.7 \mathrm{~K}, M=3.42$, 10 different shots.

Figure 8. Example traces showing repeatability of each type of laser perturbation measurement. All measurements taken on centerline.

The time during which the probe is held steady at each location is converted to a physical distance using the formula

$$
\Delta z=u_{\infty} t=M_{\infty} \sqrt{\gamma R T_{1}} t
$$

where $\Delta z$ is the relative distance between the probe and the perturbation, $u_{\infty}$ is the freestream velocity, $t$ is the time after each laser pulse, $M_{\infty}$ is the Mach number, $T_{1}$ is the static freestream temperature, $\gamma$ is the ratio of specific heats, and $R$ is the gas constant.

Figure 9 shows an example time trace from a deflectometry measurement. The time trace shows an ensemble average of the measured deflection on the y-axis. A laser pulse occurs at $t=0$. The lower horizontal axis shows the time after a laser pulse in microseconds. The upper horizontal axis shows the distance corresponding to the passage of time after the laser pulse, as given by Equation 6 .

The time traces from a single survey can be combined to form a picture of the perturbation, as in Figure 10. This plot shows the time traces at each $y$-station in the survey, offset by an amount proportional to the $y$-location of the measurement. Again, the bottom horizontal axis shows the time after the laser 


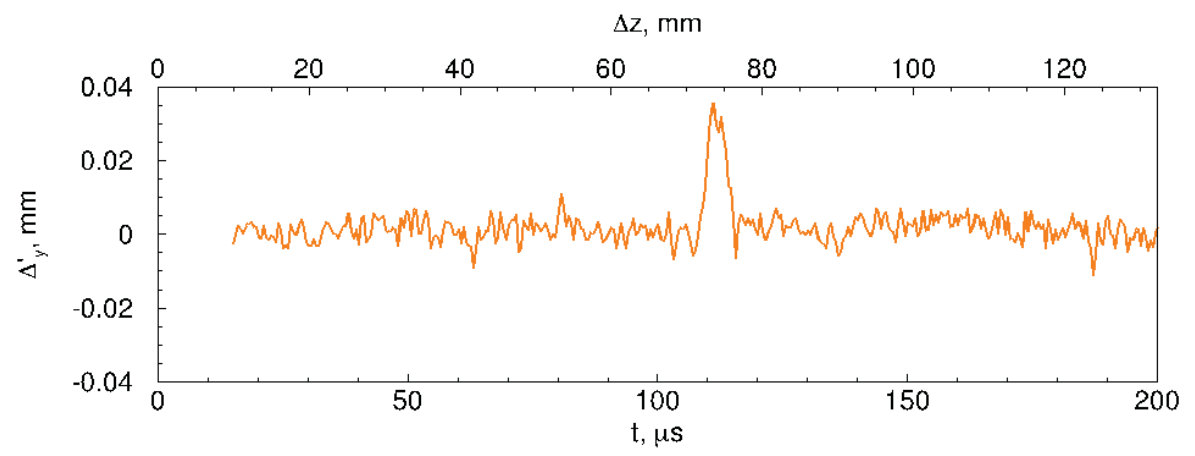

Figure 9. Example deflectometry measurement at $p_{0}=96.7 \mathbf{k P a}, T_{0}=310.9 \mathbf{K}, z=110 \mathbf{m m}$.

pulse in microseconds while the top shows the relative distance from where the perturbation is generated (Equation 6). To make a contour plot, the time traces at each location are compiled and displayed as in Figure 11. Bottom-to-top and top-to-bottom surveys were taken to ensure that these plots were repeatable. No significant variation was seen between these two types of surveys.

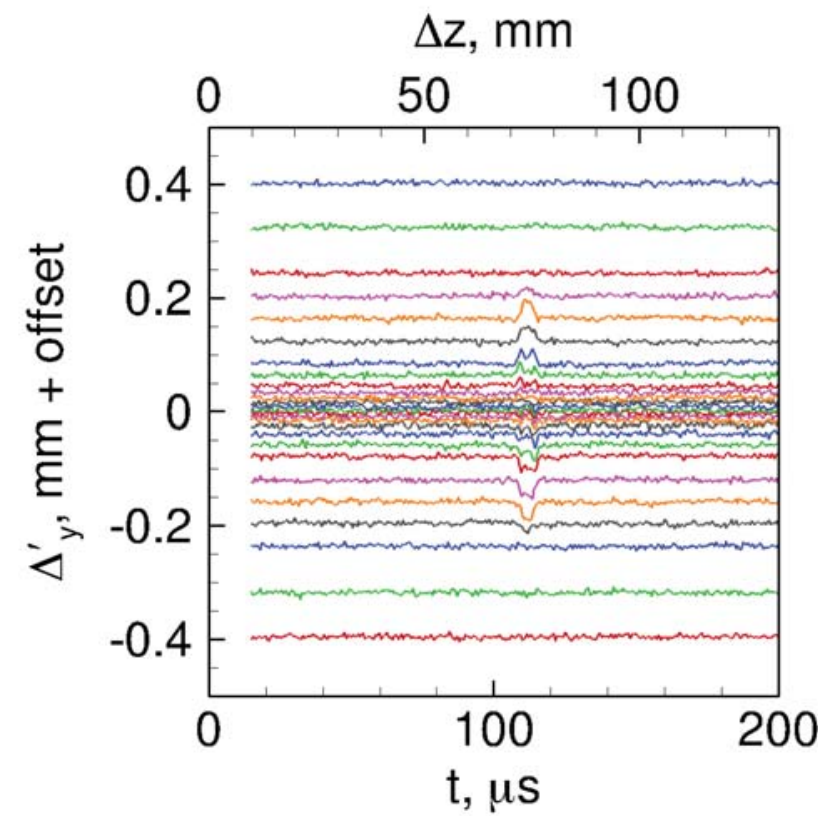

Figure 10. Compilation of example deflectometry measurements in a survey at $p_{0}=96.7 \mathrm{kPa}, T_{0}=310.9 \mathrm{~K}$, $z=110 \mathrm{~mm}$.

\section{Baseline Flow Quality of the PCT}

\section{A. Spatial Uniformity of Physical Quantities in the PCT}

Hot-wire (mass flux) measurements were made in the PCT to determine the uniformity of the flow. No laser perturbations were created in the freestream for these tests. Plots of the normalized mean mass flux for four survey locations at a stagnation pressure of $p_{0}=84.6 \mathrm{kPa}$ are provided in Figure 12. This plot is similar to the data found at other conditions. The densities in the PCT are slightly higher than originally expected because the stagnation pressures calculated in Table 2 assumed a Mach number of 3.5. The Mach number in the open jet is slightly lower at around 3.4. Figure 12 shows that the mean mass flux is uniform within $\pm 20 \mathrm{~mm}$ from the tunnel centerline. In this region, the mass flux does not vary more than $\pm 5 \%$ from the nominal value. 


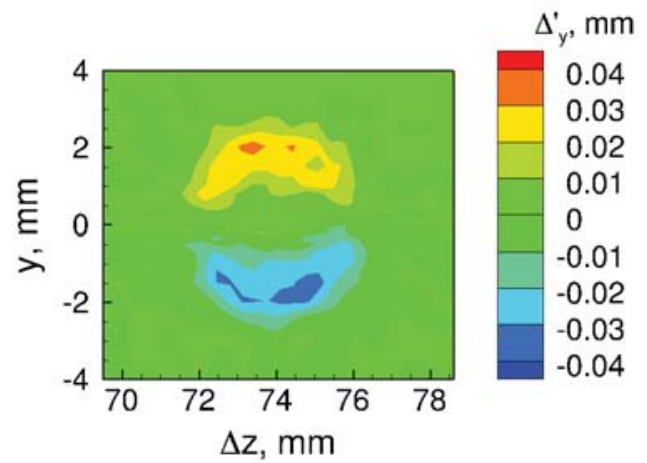

Figure 11. Contour of example deflectometry measurements in a survey at $p_{0}=96.7 \mathrm{kPa}, T_{0}=310.9 \mathrm{~K}$, $z=110 \mathrm{~mm}$.

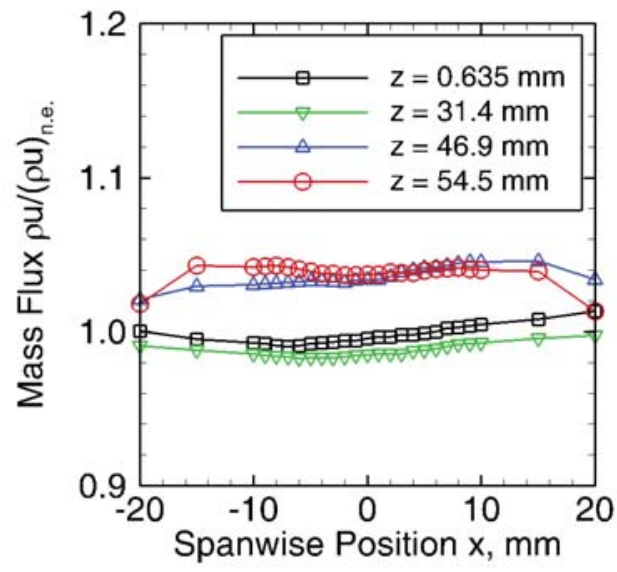

Figure 12. Mean mass flux measurements of PCT with Mach 3.5 nozzle and no forced perturbations. $p_{0}=$ $84.6 \mathrm{kPa}, T_{0}=311.4 \mathrm{~K}, \rho_{\infty}=0.046 \mathrm{~kg} / \mathrm{m}^{3}$.

The mass flux decreases towards the spanwise edges of the measurement region at the most aft measurement locations. The jet is under-expanded, so this decrease likely indicates the effect of the expansion fan. The mass flux also appears to increase with downstream location. This may be due to some small entrainment of fluid into the shear layer at the edge of the jet.

\section{B. Fluctuations in the PCT}

The freestream disturbance levels at the tested conditions can be determined by looking at the mass flux fluctuations from the hot wire measurements. However, in looking at an example of the power spectra of these baseline hot wire measurements, most of the mass flux measurements are dominated by the electronic noise from the anemometer. Figure 13(a) shows the spectra for the four conditions tested, taken on the centerline, $47 \mathrm{~mm}$ from the nozzle exit. Figure 13(b) shows the spectra for the four streamwise measurement locations, also taken on the centerline, for a stagnation pressure of about $p_{0}=84.6 \mathrm{kPa}$. The power spectral density of the mass flux over the nominal (nozzle-exit) mass flux is plotted on a logarithmic scale on the $y$-axis and the frequency in $\mathrm{Hz}$ is plotted logarithmically on the $x$-axis. The roll-off created by the analog anti-alias filter is shown at $200 \mathrm{kHz}$. The electronic noise appears to dominate the spectra from 1-100 kHz. However, the RMS fluctuations in the range of $100 \mathrm{~Hz}-1000 \mathrm{~Hz}$ show a significant rise above the electronic noise, which increases as the freestream conditions increase. The RMS fluctuations in this range are fairly small, on the order of about $0.01 \%$ at most. These small RMS fluctuations are similar to those measured in the NASA Langley Supersonic Low-Disturbance Tunnel, a quiet Mach-3.5 tunnel. ${ }^{31}$ In fact, these power spectra show measurements similar to those shown in Reference 31 from a Mach-3.5 quiet tunnel. 


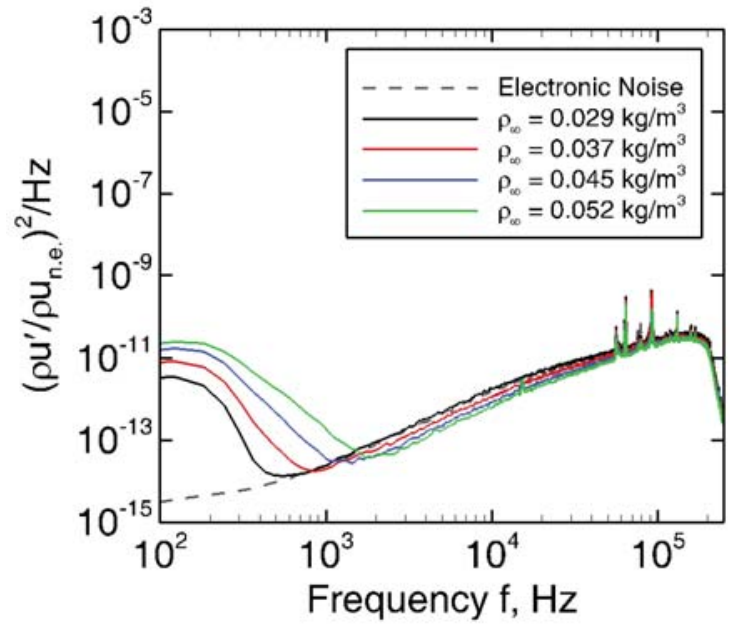

(a) $z=47 \mathrm{~mm}$, all stagnation conditions.

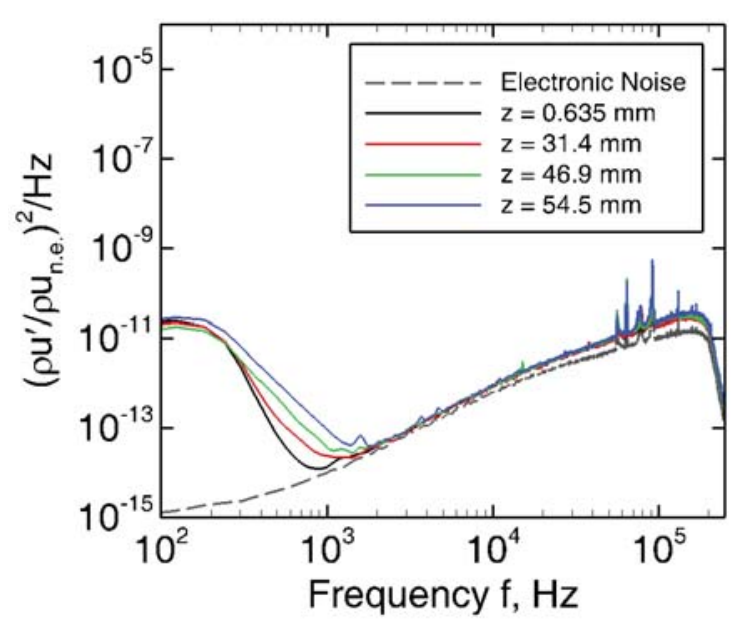

(b) $p_{0}=84.6 \mathrm{kPa}, T_{0}=311.4 \mathrm{~K}$, all $z_{\text {meas }}$ locations

Figure 13. Example spectra of baseline flow measurements, taken on the centerline of the PCT.

Further evidence that the perturbations in the PCT at these conditions are close to those seen in a quiet tunnel is in the measurement of turbulent spots. When the boundary layer on the nozzle wall is transitional, it develops turbulent spots, which can radiate acoustic noise into the freestream. Probes in the flow can measure the effects of the passing turbulent spots. An example trace of such a measurement with a hot wire is shown in Figure 14. The boundary layer on the nozzle wall is likely laminar, up to about $t=0.9985 \mathrm{~s}$, since the hot wire shows little fluctuation. Turbulent spots likely develop just afterward, showing up in the hot wire trace as five large spikes in mass flux in Figure 14. After the passage of these turbulent spots, the flow returns to the low fluctuation levels seen previously.

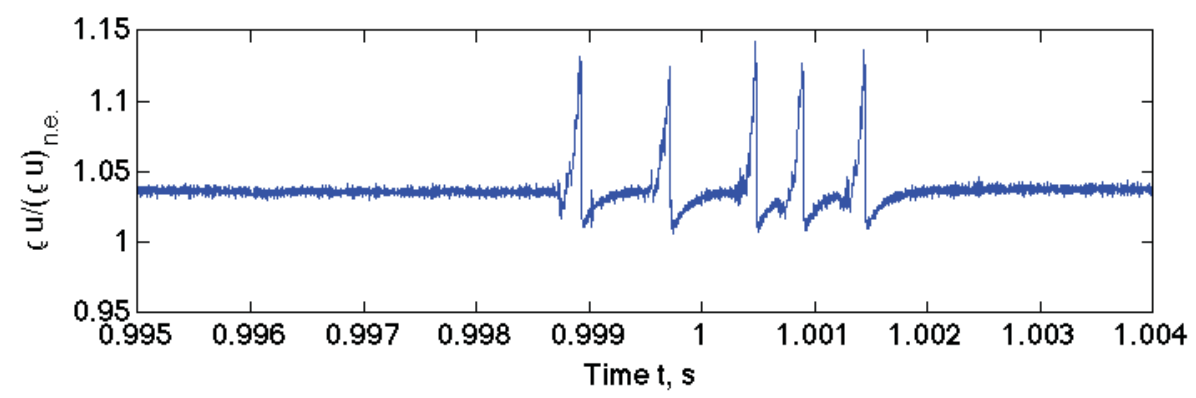

Figure 14. Example trace from hot wire of measurements of turbulent spots in the PCT. $p_{0}=201.9 \mathrm{kPa}$, $T_{0}=310.5 \mathrm{~K}, \operatorname{Re} / m=11.41 \times 10^{6} / \mathbf{m}, z=54.5 \mathrm{~mm}, M=3.42$.

Figure 15 shows time traces from four different surveys taken at similar stagnation conditions. Each time trace is from a measurement taken on the centerline at each of the specified distances from the nozzle exit. The intermittent spikes seem to be caused by acoustic noise radiated from turbulent spots on the nozzle wall. Small variations in temperature and pressure appear to change the number of turbulent spots that are measured by the hot wire. This indicates that the boundary layer on the nozzle wall is likely transitional at this condition. The RMS mass flux between $100-1000 \mathrm{~Hz}$ is about $0.2-0.3 \%$ for the conditions shown in Figure 15. Since this condition is much higher than the ones tested for the perturbation, it is likely that the nozzle-wall boundary layer at the tested conditions is laminar. 


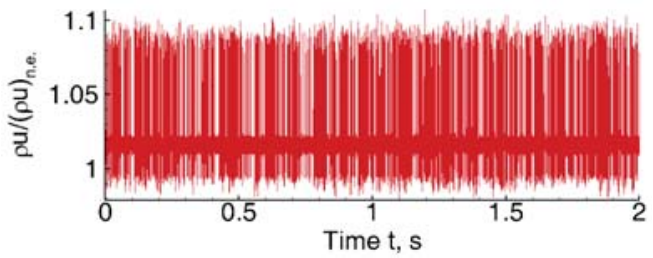

(a) $p_{0}=201.5 \mathrm{kPa}, T_{0}=310.7 \mathrm{~K}, \mathrm{Re} / m=11.37 \times$ $10^{6} / \mathrm{m}, z=0.635 \mathrm{~mm}$.

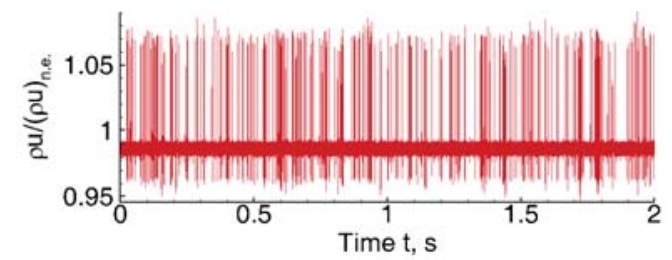

(c) $p_{0}=199.0 \mathrm{kPa}, T_{0}=310.5 \mathrm{~K}, R e / m=11.24 \times$ $10^{6} / \mathrm{m}, z=31.4 \mathrm{~mm}$.

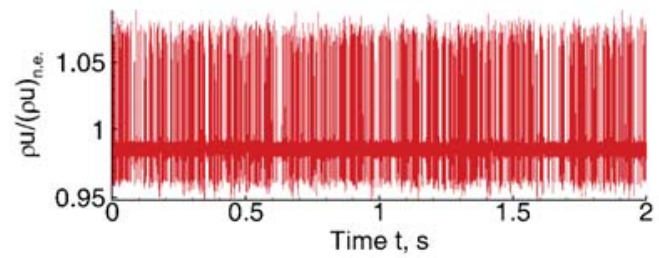

(b) $p_{0}=200.4 \mathrm{kPa}, T_{0}=310.6 \mathrm{~K}, \mathrm{Re} / m=11.32 \times$ $10^{6} / \mathrm{m}, z=31.4 \mathrm{~mm}$.

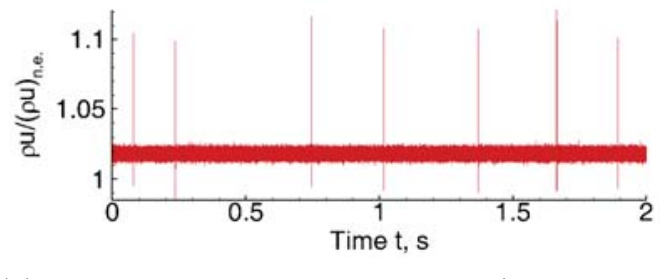

(d) $p_{0}=199.3 \mathrm{kPa}, T_{0}=311.5 \mathrm{~K}, R e / m=11.21 \times$ $10^{6} / \mathrm{m}, z=46.9 \mathrm{~mm}$.

Figure 15. Single point measurements with a hot wire on the centerline of the tunnel, organized by decreasing unit Reynolds number.

\section{Profiles of the Laser-Generated Perturbation}

In the following measurements, a perturbation is generated in the freestream. The perturbation is generated at a location $z_{\text {perturb }}$. This distance is measured from the nozzle exit when discussing measurements in the PCT. This distance is measured from the throat of the BAM6QT when discussing measurements in the BAM6QT. The streamwise location at which surveys were taken is given as $z_{\text {meas }}$. The relative distance between the measurement location and the initial location of the perturbation may be given as $\Delta z_{i}$.

\section{A. Measurements in the PCT}

Preliminary measurements with a hot wire were made using a modified lens system. Only a single YAP lens was used to create a laser perturbation at a location of $z_{\text {perturb }} \approx 30 \mathrm{~mm}$. Data were collected at each spanwise location by traversing the probe from the $-x$-direction to the $+x$-direction. The probe paused at 0.5 -mm increments for 50 shots.

The hot wires were calibrated for mass flux at a high overheat ratio of between 0.75 and 0.80 . At high overheat ratios, hot wires are more sensitive to mass flux than total temperature, as long as the mass flux and total temperature fluctuations are on the same order of magnitude. ${ }^{32}$ Schmisseur estimates that the change in temperature created by the perturbation is on the order of $100 \mathrm{~K} .{ }^{18}$ The thermal perturbation is so large that the calibrations are no longer valid unless the temperature can somehow be accounted for in the hot-wire response. Thus, only the raw voltages were used for analysis.

Figure 16 shows four contour plots for conditions that match densities seen in the BAM6QT (Table 2). The data were converted to a normalized voltage fluctuation $E_{0}^{\prime} / E_{0 \text {, mean }}$ for these plots in order to compare between the different conditions more easily. These plots all show a teardrop-shaped thermal perturbation surrounded by a nearly circular acoustic perturbation. This teardrop shape is a result limited frequency response. This sensor is not capable of fully resolving the quick passage of the thermal perturbation. Improved sensors are needed to better resolve the streamwise extent of the perturbation.

As the freestream density decreases, the perturbation decreases in magnitude and size. Figure 16(a) shows a perturbation which is about $6 \mathrm{~mm}$ wide and shows a large voltage deficit in the center of the perturbation. Figure 16(d) shows a perturbation which is about $2 \mathrm{~mm}$ wide and has a smaller voltage deficit in the center.

Density contours of the laser-generated perturbation are shown in Figure 17. These contours were created from the deflectometry measurements using the steps discussed in Section III.A.2. Three different stagnation conditions at the farthest forward and farthest aft $z_{\text {meas }}$ locations are given in these plots. For all of the data shown here, the perturbation was created on the nozzle centerline at a location of $z_{\text {perturb }}=35.6 \mathrm{~mm}$. 


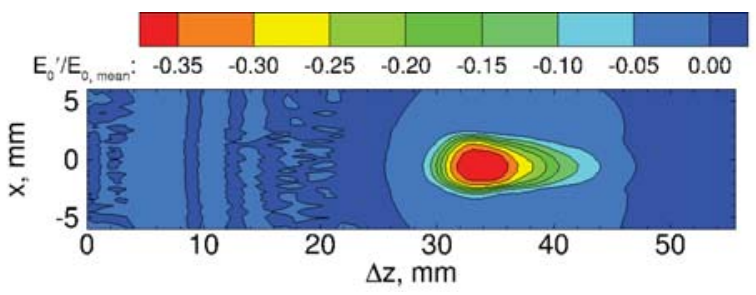

(a) $p_{0}=95.8 \mathrm{kPa}, T_{0}=311.1 \mathrm{~K}, \rho_{\infty}=0.052 \mathrm{~kg} / \mathrm{m}^{3}$.

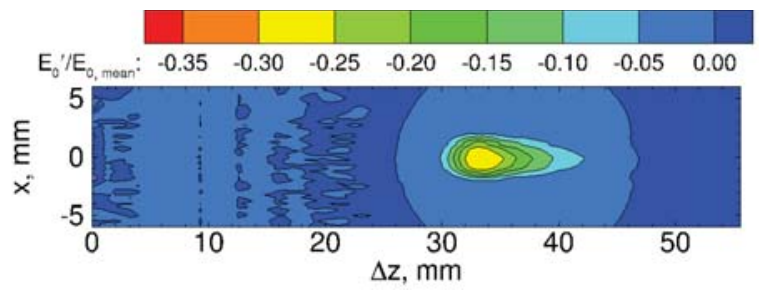

(c) $p_{0}=68.9 \mathrm{kPa}, T_{0}=311.1 \mathrm{~K}, \rho_{\infty}=0.038 \mathrm{~kg} / \mathrm{m}^{3}$.

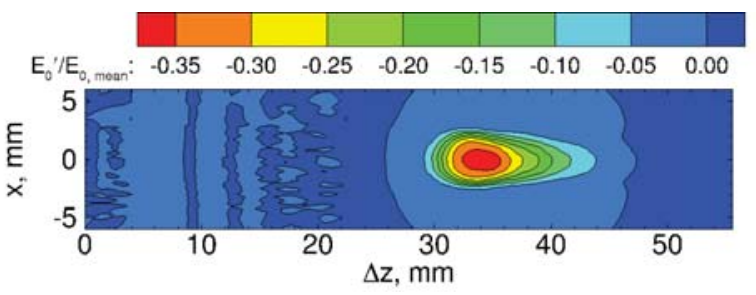

(b) $p_{0}=86.2 \mathrm{kPa}, T_{0}=310.9 \mathrm{~K}, \rho_{\infty}=0.047 \mathrm{~kg} / \mathrm{m}^{3}$.

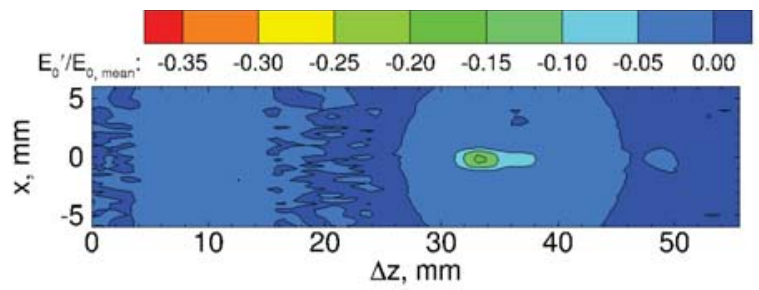

(d) $p_{0}=51.6 \mathrm{kPa}, T_{0}=311.6 \mathrm{~K}, \rho_{\infty}=0.028 \mathrm{~kg} / \mathrm{m}^{3}$.

Figure 16. Contour plots of normalized voltage fluctuation, created from hot wire measurements made in the PCT $\left(M_{\infty} \approx 3.42, \Delta z_{i} \approx 30 \mathbf{m m}\right)$. 50 shots averaged.

The density contours in Figure 17 show a roughly elliptical or circular shape. Thus, the optical technique is fast enough to resolve the passage of the perturbation in the streamwise direction. This frequency response is promising, but the measurement technique has other limitations. As the change in density at the center of the perturbation decreases, the general signal-to-noise ratio decreases. Furthermore, in order to apply the inverse Abel transform to the deflectometry measurements, an assumption of axisymmetry had to be applied.

Line cuts from these contour plots can also be taken to yield density profiles as shown in Figure 18. Figure 18(a) shows the variation in density profiles when surveys are taken at different distances from the perturbation. Figure 18(b) shows the variation in density profiles when the freestream stagnation pressure is varied. There is a large amount of noise in the center of the profile for the cases where the density change is small (e.g., for the lowest stagnation pressure in Figure 18(b) or the greatest $z_{\text {meas }}$ in Figure 18(a)). When the perturbation is weaker, the signal-to-noise ratio becomes poorer. This noise is then carried through the inverse Abel transform to produce the large noise at the center of the perturbation's density profile.

Measurements taken closer to the perturbation at $z_{\text {meas }}=90 \mathrm{~mm}$ at the highest stagnation pressures yield density profiles across the perturbation that do not seem realistic. For example, Figure 18(a) shows the different conditions tested in the PCT at the survey location of $z_{\text {meas }}=90 \mathrm{~mm}$. At the highest stagnation pressure shown $\left(p_{0}=96.6 \mathrm{kPa}\right)$, the density profile yields a negative density. The next highest stagnation pressure shown $\left(p_{0}=86.4 \mathrm{kPa}\right)$, the density profile yields a near-zero density at the center of the perturbation. It is possible that this type of density profile is caused by the presence of free electrons and ions when the perturbation is large (at higher stagnation conditions) or when measurements are taken close to the perturbation generation site (at lower $z_{\text {meas }}$ ). At the most upstream survey location of $z_{\text {meas }}=90 \mathrm{~mm}$, about $80 \mu$ s have passed since the laser perturbation was created. At the most downstream survey location of $z_{\text {meas }}=150 \mathrm{~mm}$, about $170 \mu$ s have passed since the laser perturbation was created. The presence of free electrons would invalidate the Gladstone-Dale assumption and reduces the confidence in the inferred density. However, the index of refraction inferred from the inverse Abel transform is still valid.

\section{B. Measurements in the BAM6QT}

The traversing probe is capable of moving vertically (in the $y$-direction) during a run, and axially/streamwise (in the $z$-direction) between runs. For the purpose of making contour plots, the probe was held in one position during the full run and only traversed in the $y$-direction between runs. This was done to average the response to as many laser pulses as possible before conditions changed too much in the BAM6QT. The profiles used in Figure 19 are compiled using 9 different runs. Each run corresponds to a single $y$-location. Ten averages of the response to a laser perturbation are taken at each $y$-location. The number of averages in the BAM6QT 


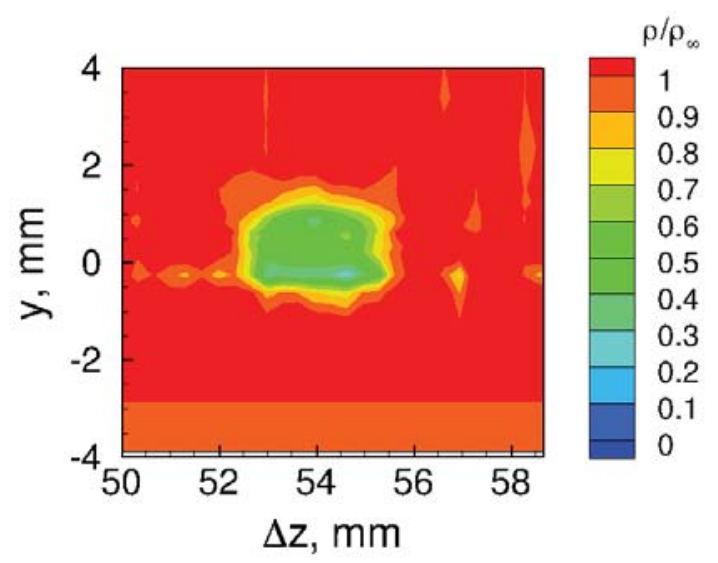

(a) $p_{0}=69.0 \mathrm{kPa}, T_{0}=311.1 \mathrm{~K}, z_{\text {meas }}=90 \mathrm{~mm}$.

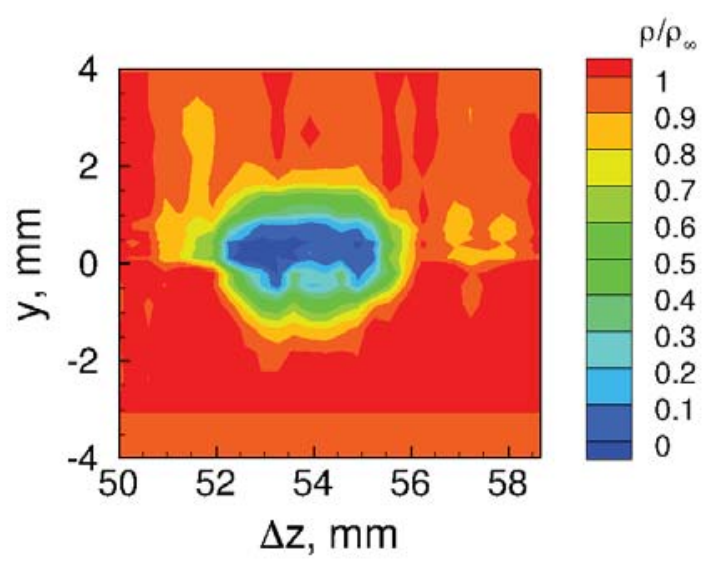

(c) $p_{0}=86.4 \mathrm{kPa}, T_{0}=311.1 \mathrm{~K}, z_{\text {meas }}=90 \mathrm{~mm}$.



(e) $p_{0}=96.6 \mathrm{kPa}, T_{0}=310.8 \mathrm{~K}, z_{\text {meas }}=90 \mathrm{~mm}$.

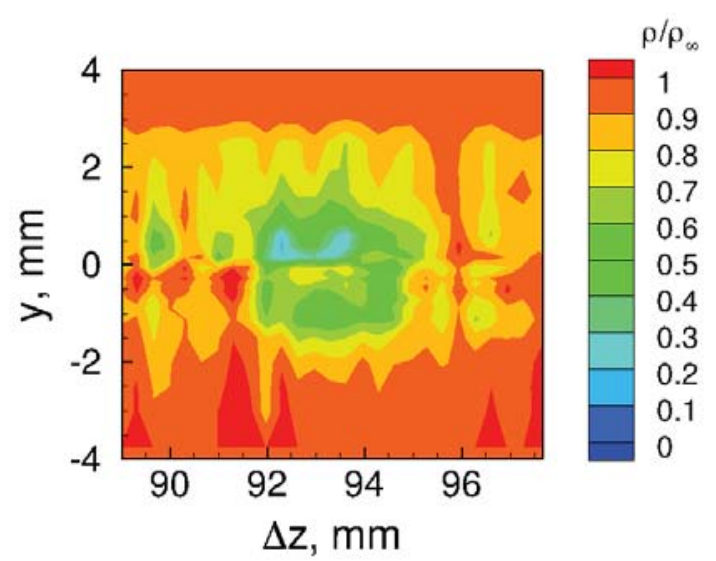

(b) $p_{0}=69.0 \mathrm{kPa}, T_{0}=311.1 \mathrm{~K}, z_{\text {meas }}=150 \mathrm{~mm}$.

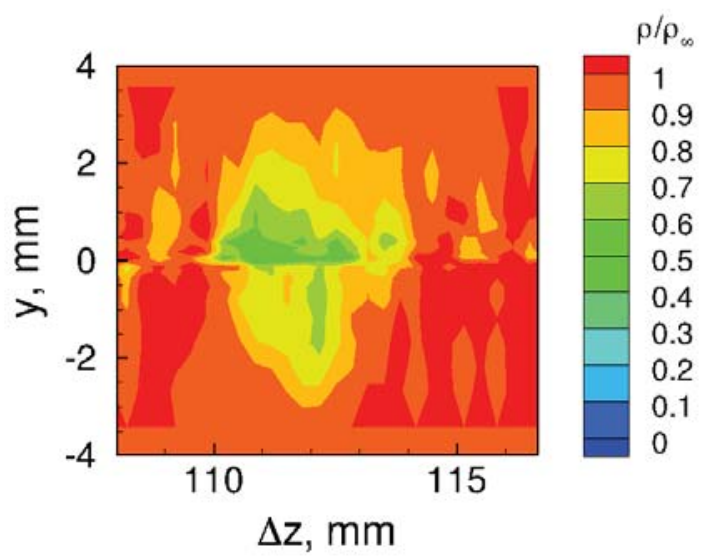

(d) $p_{0}=86.2 \mathrm{kPa}, T_{0}=311.0 \mathrm{~K}, z_{\text {meas }}=150 \mathrm{~mm}$.



(f) $p_{0}=96.7 \mathrm{kPa}, T_{0}=311.2 \mathrm{~K}, z_{\text {meas }}=150 \mathrm{~mm}$.

Figure 17. A comparison of density contour plots for the laser-generated perturbation, created from deflectometry measurements made in the PCT $\left(M_{\infty} \approx 3.42\right) .150$ shots averaged. 



(a) $p_{0} \approx 97 \mathrm{kPa}, T_{0} \approx 311 \mathrm{~K}, \rho_{\infty} \approx$ $0.05 \mathrm{~kg} / \mathrm{m}^{3}$, different measurement positions.
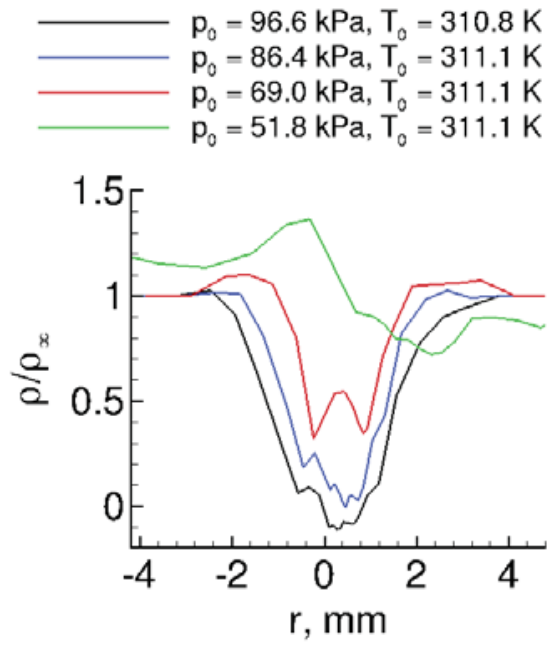

(b) Different freestream conditions with surveys taken at $z_{\text {meas }}=90 \mathrm{~mm}$.

Figure 18. A comparison of density contour plots for the laser-generated perturbation, created from deflectometry measurements made in the PCT $\left(M_{\infty} \approx 3.42\right) .150$ shots averaged.

is greatly reduced from that of the PCT due to the tunnel's shorter run time.

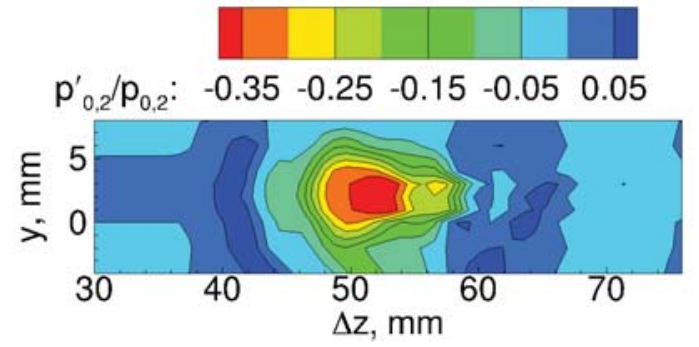

(a) $p_{0} \approx 1092 \mathrm{kPa}, T_{0} \approx 428 \mathrm{~K}, \rho_{\infty}=0.040 \mathrm{~kg} / \mathrm{m}^{3}$, $\Delta z_{i}=34.0 \mathrm{~mm}$.

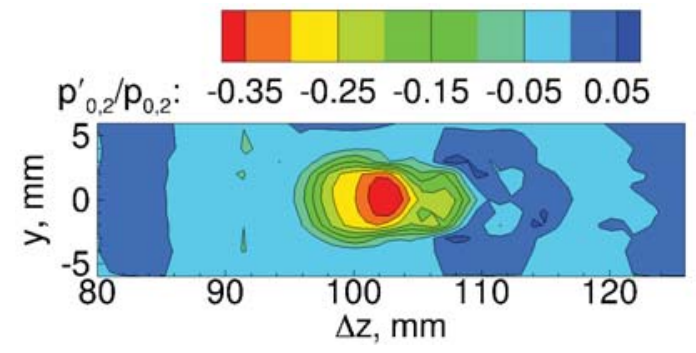

(b) $p_{0} \approx 1092 \mathrm{kPa}, T_{0} \approx 428 \mathrm{~K}, \rho_{\infty}=0.040 \mathrm{~kg} / \mathrm{m}^{3}$, $\Delta z_{i}=87.5 \mathrm{~mm}$.

Figure 19. A comparison of contour plots, created from pitot Kulite measurements in the BAM6QT $\left(M_{\infty} \approx 6.0\right)$.

The frequency response of a Kulite XCQ sensor with a B-screen is said to be flat only up to about $20 \%$ of its resonant frequency. ${ }^{36,37}$ The Kulite sensors mounted in the probes have a resonant frequency of about $330 \mathrm{kHz}$. Thus, the frequency response is expected to be about $60 \mathrm{kHz}$, which is lower than that of the hot wire. However, the Kulite sensor is more robust, and thus, easier to use for measurements in the BAM6QT. Measurements of the laser-generated perturbation in the BAM6QT show a similar shape to those made with hot wires in the PCT. The shape of the thermal disturbance is more of an elongated ellipse rather than a circle, due to the limited frequency response. The weak shock wave that emanates from the thermal core can also be observed as a blue circle surrounding the thermal core. This shock wave is stronger when the probe is placed closer to the perturbation, as in Figure 19(a). When the probe is farther downstream from the location of perturbation generation, the shock wave has a larger radius and is weaker (Figure 19(b)). The perturbation magnitude does not appear to change very much between the two different measurement locations. The deficit in pitot pressure is large and remains at roughly $35 \%$ at the center of the perturbation.

Similar measurements made with a PCB probe in the BAM6QT are shown in Figures 20 and 21. Figure 20 shows an ensemble average of the measured response to the perturbation at each survey point, offset by an amount proportional to the $y$-location. Variation in the time of arrival is seen in each trace. Since each $y$-location responds to a different run in the tunnel, this jitter may be related to the variation in total temperature from run to run.

Despite the jitter from shot to shot, Figure 21 shows a perturbation that is more circular than previous 


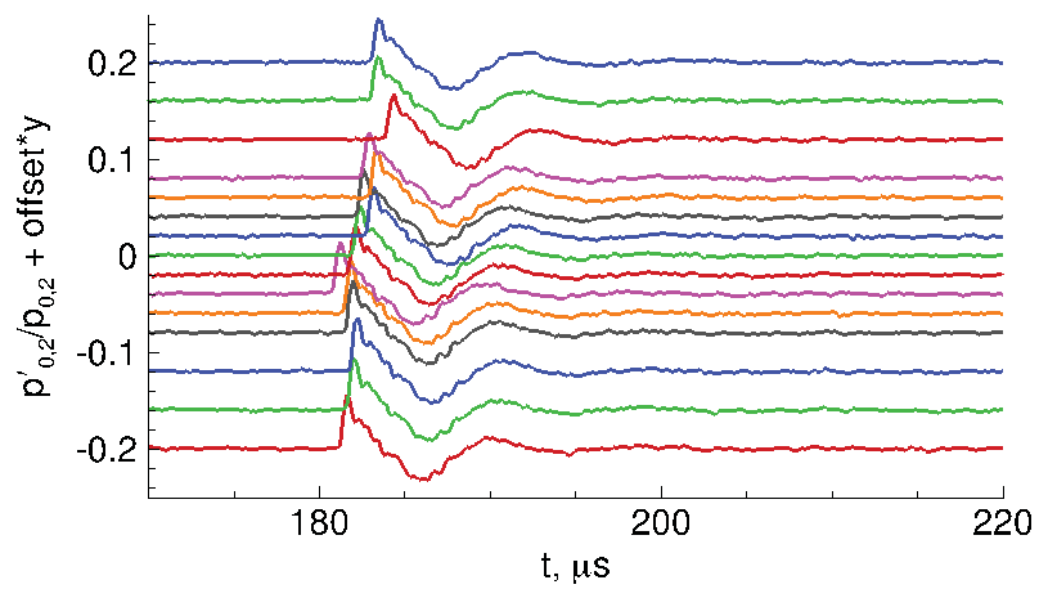

Figure 20. Line plot of a laser-generated perturbation, offset by an amount proportional to the spanwise location of the measurement in the tunnel. $M=6.0, p_{0}=1022 \mathrm{kPa}, T_{0}=139.6^{\circ} \mathbf{C}, R e / \mathrm{m}=11.3 \times 10^{6} / \mathbf{m}$, $\rho_{\infty}=0.043 \mathrm{~kg} / \mathrm{m}^{3}$.

measurements. The perturbation appears to have a diameter of about $6 \mathrm{~mm}$ at the conditions shown. The PCB sensor has a sufficient frequency response to resolve the streamwise extent of the perturbation. Thus, the diameter of the perturbation in the $z$-direction appears to be roughly the same as the $y$-direction.

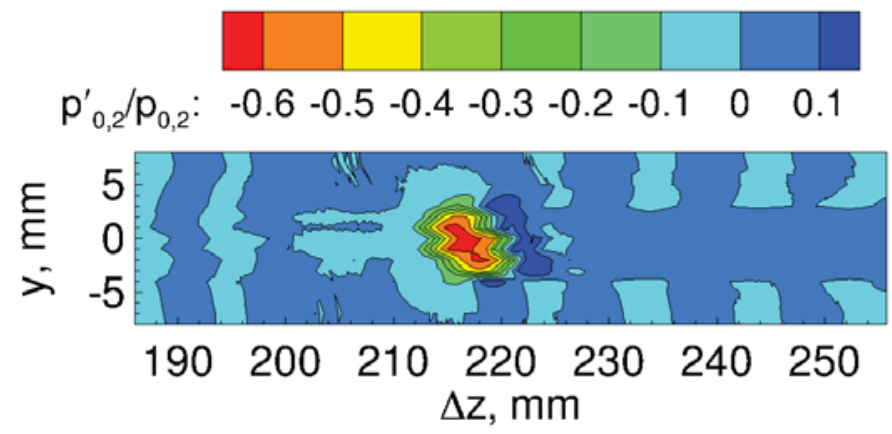

Figure 21. Contour plot of a laser-generated perturbation, compiled from measurements made with a PCB probe at $M=6.0, p_{0}=1022 \mathbf{k P a}, T_{0}=139.6^{\circ} \mathbf{C}, R e / m=11.3 \times 10^{6} / \mathbf{m}, \rho_{\infty}=0.043 \mathrm{~kg} / \mathbf{m}^{3}$.

The shape of this perturbation in the contour plot is very irregular. This could be a result of the large pitot probe size or of the variation in temperature from run to run. The stagnation temperature of the traces that make up the contour plot have an average of $T_{0}=139.6^{\circ} \mathrm{C}$. However, each time trace (constant $y$ station) can be as much as $\pm 5^{\circ} \mathrm{C}$ off of this average. This change in temperature may affect the convection time of the perturbation and create jitter in each of the survey points.

The magnitude of the perturbation, as measured by the PCB, also appears to be much greater than the already large magnitude measured by the Kulite sensor. This could be due to a number of reasons. The measurement with the PCB probe uses the factory-provided calibration and the inference of the pitot pressure from the freestream conditions. A dynamic calibration of the PCB sensor was not available at the time. The Kulite sensor may also have too low a frequency response to catch the full deficit in pitot pressure. Furthermore, the response of these pressure transducers to a thermal perturbation may not be simple. Both Kulite and PCB transducers detect the pressure perturbation induced by the transit of the thermal perturbation through the probe bow shock, and the transfer function associated with that interaction has not been quantified. 


\section{Summary}

A local thermal perturbation generated by a pulsed laser was characterized for receptivity studies. Characterization measurements were conducted in two wind-tunnel facilities. The NASA Langley PCT was operated at Mach 3.5 in continuous flow, allowing for detailed surveys of the perturbation. Measurements in this facility were made with a hot wire probe and a deflectometry method. The Purdue BAM6QT is a Mach-6 facility which has a short run time, but is capable of running with low freestream acoustic noise at high Reynolds numbers. Measurements in the BAM6QT were made with pitot probes using fast pressure transducers.

The perturbation size and magnitude change with the freestream conditions. At the highest density, in both tunnels, the perturbation is about $6 \mathrm{~mm}$ in diameter. At the lowest density tested in the PCT, the perturbation is about $2 \mathrm{~mm}$ in diameter. The measurement techniques employed here imply a large amplitude, ranging from $35-80 \%$ of the freestream conditions. Many uncertainties are associated with these techniques, so these amplitudes are considered to be qualitative. The streamwise extent of the perturbation is capable of being resolved with high frequency measurements, such as with an optical technique. Limitations exist in the measurement technology used and will require improvement to make higher fidelity measurements.

\section{Future Work}

A similar laser-generated perturbation will be used with a 3-m-circular-arc flared cone model. The effect of the laser perturbation on the boundary layer instabilities of this model will be investigated. The instabilities in the boundary layer of this model will be measured with surface-mounted PCB fast pressure transducers. Spanwise measurements of these instabilities will show whether the perturbation generated in the freestream generates an axisymmetric disturbance in the boundary layer. Streamwise measurements of these instabilities will show how the freestream perturbation affects the growth of boundary layer disturbances. These measurements will provide additional insight into receptivity and ultimately aid in the development and improvement of amplitude-based transition prediction methods.

\section{Acknowledgments}

This research was funded by AFOSR Grant FA9550-12-1-0167 and the NASA Pathways Intern Employment Program. Special thanks are also given here to Ricky Clark and Rhonda Mills for their help in running the facilities at NASA Langley. Paul Danehy and Steve Wilkinson at NASA Langley Research Center are also thanked for their guidance on this project. Steven Collicott at Purdue University designed the perturbation-forming optics for this project.

\section{References}

\footnotetext{
${ }^{1}$ M. V. Morkovin. Critical evaluation of transition from laminar to turbulent shear layers with emphasis on hypersonically traveling bodies. Technical report AFFDL-TR-68-149, Air Force Flight Dynamics Laboratory, Wright-Patterson Air Force Base, OH 45433, Mar 1969.

${ }^{2}$ L. M. Mack. Linear stability theory and the problem of supersonic boundary-layer transition. AIAA Journal, 13(3):278289, Mar 1975.

${ }^{3}$ J. M. Kendall. Wind tunnel experiments relating to supersonic and hypersonic boundary-layer transition. AIAA Journal, 13(3):290-299, Mar 1975.

${ }^{4} \mathrm{M}$. V. Morkovin. Note on the assessment of flow disturbances at a blunt body traveling at supersonic speeds owing to flow disturbances in free stream. Journal of Applied Mechanics, 27(2):223-229, Jun 1960.

${ }^{5}$ P. Balakumar. Receptivity of a supersonic boundary layer to acoustic disturbances. AIAA Journal, 47(5):1069-1078, 2009.

${ }^{6} \mathrm{P}$. Balakumar. Receptivity of hypersonic boundary layers to distributed roughness and acoustic waves. AIAA Paper 2013-0082, Jan 2013.

${ }^{7}$ P. Balakumar and M. A. Kegerise. Receptivity of hypersonic boundary layers to acoustic and vortical disturbances. AIAA Paper 2011-0371, Jan 2011.

${ }^{8}$ A. V. Fedorov and M. V. Kozlov. Receptivity of high-speed boundary layer to solid particulates. AIAA Paper 2011-3925, Jun 2011.

${ }^{9}$ K. Mahesh, S. K. Lele, and P. Moin. The influence of entropy fluctuations on the interaction of turbulence with a shock wave. Journal of Fluid Mechanics, 334:353-379, 1997.
} 
${ }^{10} \mathrm{Y}$. Huang and X. Zhong. Numerical study of freestream hot-spot perturbation on boundary-layer receptivity for blunt compression-cones in Mach-6 flow. AIAA Paper 2011-3078, Jun 2011.

${ }^{11}$ J. M. Kendall. Boundary layer receptivity to freestream turbulence. AIAA Paper 1990-1504, Jun 1990.

${ }^{12} \mathrm{M}$. Wiegel and R. W. Wlezien. Acoustic receptivity of laminar boundary layers over wavy walls. AIAA Paper 1993-3280, Jul 1993.

${ }^{13}$ A. A. Maslov, A. N. Shiplyuk, A. A. Sidorenko, and D. Arnal. Leading-edge receptivity of a hypersonic boundary layer on a flat plate. Journal of Fluid Mechanics, 426:73-94, 2001.

${ }^{14}$ J. D. Schmisseur, S. H. Collicott, and S. P. Schneider. Laser-generated localized freestream perturbations in supersonic and hypersonic flows. AIAA Journal, 38(4), Apr 2000.

${ }^{15}$ T. R. Salyer. Laser Differential Interferometry for Supersonic Blunt Body Receptivity Experiments. PhD thesis, School of Aeronautics \& Astronautics, Purdue University, West Lafayette, IN, May 2002.

${ }^{16}$ D. Heitmann, R. Radespiel, and Christian Kähler. Investigation of the response of a hypersonic boundary layer to controlled acoustic disturbances. AIAA Paper 2010-0536, Jan 2010.

${ }^{17}$ J. B. McGuire. Fluid dynamic perturbations using laser induced breakdown. Master's thesis, Purdue University, West Lafayette, IN, August 1994.

${ }^{18}$ J. D. Schmisseur. Receptivity of the Boundary Layer on a Mach-4 Elliptic Cone to Laser-Generated Localized Freestream Perturbations. PhD thesis, Purdue University, West Lafayette, IN, December 1997.

${ }^{19}$ D. Heitmann and R. Radespiel. Simulation of the interaction of a laser generated shock wave with a hypersonic conical boundary layer. AIAA Paper 2011-3875, Jun 2011.

${ }^{20}$ D. Heitmann, R. Radespiel, and H. Knauss. Experimental study of Mach 6 boundary layer response to laser generated disturbances. AIAA Paper 2011-3875, Jun 2011.

${ }^{21}$ D. Heitmann. Transitionsuntersuchungen in hypersonischen Grenzschichten mit laserinduzierten Störungen. PhD thesis, Institüt fur Strömungsmechanik (Institute of Fluid Mechanics), Technische Universität Braunschweig, Braunschweig, Germany, 2011.

${ }^{22}$ S. P. Schneider. Effects of high-speed tunnel noise on laminar-turbulent transition. Journal of Spacecraft and Rockets, 38(3):323-333, May-Jun 2001. 2008.

${ }^{23}$ S. P. Schneider. Development of hypersonic quiet tunnels. Journal of Spacecraft and Rockets, 45(4):641-664, Jul-Aug

${ }^{24}$ A. Chou, B. M. Wheaton, C. A.C. Ward, P. L. Gilbert, L. E. Steen, and S. P. Schneider. Instability and transition research in a Mach-6 quiet tunnel. AIAA Paper 2011-0283, Jan 2011.

${ }^{25}$ L. E. Steen. Characterization and development of nozzles for a hypersonic quiet wind tunnel. Master's thesis, School of Aeronautics \& Astronautics, Purdue University, West Lafayette, IN, Dec 2010.

${ }^{26}$ A. Chou, S. P. Schneider, and S. H. Collicott. Measurements of the interaction of an upstream laser perturbation with a forward-facing cavity. AIAA Paper 2013-0391, Jan 2013.

${ }^{27}$ D. C. Berridge, A. Chou, C. A.C. Ward, P. L. Gilbert, L. E. Steen, T. J. Juliano, S. P. Schneider, and J. E. Gronvall. Hypersonic boundary-layer transition experiments in a Mach-6 quiet tunnel.

${ }^{28}$ V. I. Eremin, L. V. Norinskii, and V. A. Pryadein. Frequency dependence of the threshold of optical breakdown in air in the ultra-violet band. JETP Letters, 13(8):307-310, 1971.

${ }^{29}$ C. Grey Morgan. Laser-induced breakdown phenomena. Scientific Progress, 65(257):31-50, Spring 1978.

${ }^{30}$ H. Yan, R. Adelgren, M. Boguszko, G. Elliott, and D. Knight. Laser energy deposition in quiescent air. AIAA Journal, 41(10), Oct 2003.

${ }^{31}$ M. A. Kegerise, L. R. Owens, and R. A. King. High-speed boundary-layer transition induced by an isolated roughness element. AIAA Paper 2010-4999, Jun 2010.

${ }^{32}$ A. J. Smits, K. Hayakawa, and K. C. Muck. Constant temperature hot-wire anemometer practice in supersonic flow. Part I: The normal wire. Experiments in Fluids, 1(2):83-92, Jun 1983.

${ }^{33} \mathrm{~S}$. McIntyre and G. Settles. Optical experiments on axisymmetric compressible turbulent mixing layers. AIAA Paper 1991-0623, Jan 1991.

${ }^{34}$ U. Kogelschatz and W. R. Schneider. Quantitative schlieren techniques applied to high current arc investigations. Applied Optics, 11(8):1822-1832, August 1972.

${ }^{35}$ Kulite Semiconductor Products. Kulite pressure transducer handbook.

${ }^{36}$ M. A. Rotea, L. A. Randall, G. Song, and S. P. Schneider. Model identification of a Kulite pressure transducer. AIAA Paper 1996-2278, Jun 1996.

${ }^{37}$ S. J. Beresh, J. F. Henfling, R. W. Spillers, and B. O. M. Pruett. Measurement of fluctuating wall pressures beneath a supersonic turbulent boundary layer. AIAA Paper 2010-0305, Jan 2010.

${ }^{38}$ A. Abney, C. Ward, D. Berridge, R. Greenwood, and S. P. Schneider. Hypersonic boundary-layer transition experiments in the Boeing/AFOSR Mach-6 Quiet Tunnel. AIAA Paper 2013-0375, Jan 2013.

${ }^{39} \mathrm{~K}$. Fujii. Experiment of the two-dimensional roughness effect on hypersonic boundary-layer transition. Journal of Spacecraft and Rockets, 43(4):731-738, 2006.

${ }^{40}$ M. Estorf, R. Radespiel, S. P. Schneider, H. B. Johnson, and S. Hein. Surface-pressure measurements of second-mode instability in quiet hypersonic flow. AIAA Paper 2009-4054.

${ }^{41}$ D. C. Berridge, K. M. Casper, S. J. Rufer, C. R. Alba, D. R. Lewis, S. J. Beresh, and S. P. Schneider. Measurements and computations of second-mode instability waves in three hypersonic wind tunnels. AIAA Paper 2010-5002, Jun 2010.

${ }^{42}$ C. R. Alba, K. M. Casper, S. J. Beresh, and S. P. Schneider. Comparison of experimentally measured and computed second-mode disturbances in hypersonic boundary layers. AIAA Paper 2010-0897, Jan 2010. 